\title{
ILUMINACIÓN Y CONFORT EN AULAS Y LABORATORIOS DE CARRERAS TÉCNICAS DE GRADO UNIVERSITARIO
}

Corvalán R., Ferrari E., Sanabria N., Titiosky V., Sáenz Pérez J., Amarilla A., Peris J., Marsilli C., Larrea D. $\left(^{*}\right)$

\section{RESUMEN}

El presente trabajo, analiza el grado de iluminación y confort, de laboratorios y aulas de carreras técnicas universitarias, de acuerdo al marco reglamentario nacional del factor de iluminación en espacios de trabajo, considerando a la actividad educativa incluida. Utilizando el protocolo aprobado por la Superintendencia de riesgos de trabajo, para la medición del factor de iluminación, tomando además las variaciones de temperatura y el factor del viento, como componentes del factor confort, necesario para una adecuada transmisión y adquisición de los contenidos didácticos.

\section{Palabras Clave:}

Iluminación, confort, energía, medición, temperatura,

\section{INTRODUCCIÓN:}

La iluminación relacionada a la educación, debe tener presente un gran número de luminarias, ya que debe abarcar espacios grandes, en consonancia con el incremento de la masividad de la educación pública, también deben poseer características especiales a las luminarias convencionales, utilizadas en viviendas, teniendo mayor potencia, brillo, incandescencia, etc.
Por ello es necesario analizar la tarea visual que se realiza y determinar el nivel de iluminación necesarias para un correcto uso del espacio, y verificar que cumplan con las normativas de seguridad y comodidad, confort.

La cantidad adecuada de luz para realizar cómodamente una tarea visual concreta, como es el estudio en aulas y laboratorios, es un requisito fundamental para una unidad educativa.

La superintendencia de Riesgos del Trabajo SRT publicó en su sitio web (www.srt. gob.ar) una guía práctica sobre iluminación. Partiendo de la guía publicada se realizó el análisis de los indicadores y método de medición estipulados en el protocolo.

El protocolo aprobado por la SRT es acompañado por una guía práctica que inicia con información teórica básica sobre luz, visión y fotometría. Respecto a los aspectos metodológicos, la técnica de relevamiento se fundamenta en una cuadrícula de puntos de medición que cubre toda la zona analizada. Se incluye un método de cálculo para estimar la cantidad de puntos de medición en base a las dimensiones geométricas del local. Se mide la iluminancia horizontal existente en el centro de cada área a la altura de 0.8 metros sobre el nivel del suelo. Esta altura recomen- 
dada corresponde a la altura estándar de plano de trabajo horizontal en oficinas.

El protocolo se presenta útil para sistematizar el análisis de iluminancias y evaluar los niveles de iluminación del espacio de trabajo horizontal. Por otro lado permite describir algunos aspectos del factor ambiental iluminación, con poco desarrollo de la iluminación natural, no considerada en muchos casos por la utilización en clases de cañones proyectores que para su uso, se oscurecen el aula, dejándola así para todas las demás clases.

La capacidad de una luminaria para dirigir la luz hacia el plano de trabajo, puede establecerse de acuerdo a su incidencia en $\operatorname{lux} / \mathrm{m} 2$, que combinado con temperatura y renovación del aire del local, dan datos sobre el confort que se analizan en esta investigación.

\section{Materiales y Métodos}

Los instrumentos utilizados fueron:

- Luxómetro Extech Modelo 401027, rango de medición de 0 a 20.000 lux, tiempo de muestreo 0,4 segundos. Resolución 1 lux
- Termómetro digital: Extech Modelo EA10 resolución $0,1^{\circ} \mathrm{C}$. Escala de medición de 0 a 200 grados centígrados

- Anemómetro Extech Modelo 45118, resolución 0,01 metros por segundo, escala 0,50 a $28 \mathrm{~m} / \mathrm{seg}$

En base a las normas de medición estándar, análisis de componentes principales, se realizaron las mediciones dentro de la cuadricula establecida, como así también se realizaron mediciones testigo fuera de la zona del cono de iluminación,

Se tomó una altura de medición de 0,75 mts, por ser el plano de trabajo, pupitres de los alumnos y profesores. Considerando que el protocolo debería ser flexible en función de las distintas alturas de puestos de trabajo.

Se calculó un valor promedio de las iluminancias del grillado que es comparado para cada sector con el valor de referencia dado por el Decreto 351/79 (Tabla 1 y Tabla 2 del Anexo IV) según la tarea visual y el tipo de edificio y local.

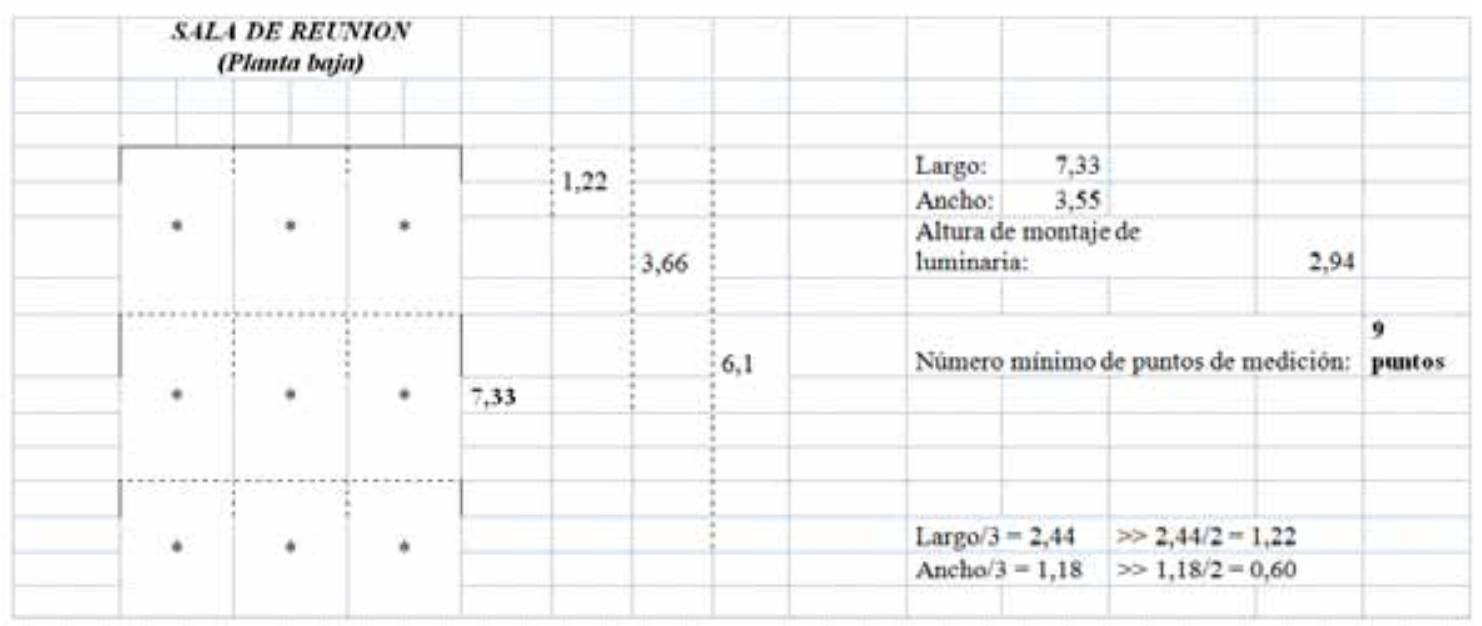


Aula pequeña - Sala de Reunión

Largo $7,33 \mathrm{~m}$ - Ancho $3,55 \mathrm{~m}$ - Altura $2,94 \mathrm{~m}$

Indice local $\mathrm{x}=\frac{l \text { argoxancho }}{\text { altura }(\text { l } \text { argo }+ \text { ancho })}=\frac{7,33 \times 3,55}{2,94(7,33+3,55)}=\frac{26,025}{31,9872}=0,81=1$

$\mathrm{X}=1 \quad$ Número de puntos de medición: $(x+2)^{2}=(1+2)^{2}=9$

$\underline{\text { Aula } 2}$ - Planta Baja

Largo $9,85 \mathrm{~m}$ - Ancho $14,68 \mathrm{~m}$ - Altura $2,96 \mathrm{~m}$

Indice local $\mathrm{x}=\frac{\text { larg oxancho }}{\text { altura }(l \text { arg } o+\text { ancho })}=\frac{144,6}{72,6}=1,99=2$

$\mathrm{X}=2$ Número de puntos de medición: $(x+2)^{2}=(2+2)^{2}=16$

Resultados:

Aula 2 - $\mathrm{Fe}-$ cha $28 / 10 / 2015$

Hora de inicio: 19 hs $30 \mathrm{~m}$; Hora de finalización: 20 hs

- Cortinas cerradas

- Ventiladores prendidos (total 9: 8 andan y el del punto $\mathrm{y}_{1}$ no anda)

\begin{tabular}{|c|c|c|}
\hline PUNTO & IUUMINACION & TEMPERATURA \\
\hline 1 & 52,9 & $25^{\circ}, 5$ \\
\hline 2 & 57,2 & $25^{\circ}, 6$ \\
\hline 3 & 54,9 & $25^{\circ}, 6$ \\
\hline 4 & 50,1 & $26^{\circ}$ \\
\hline 5 & 62,2 & $25^{\circ}, 7$ \\
\hline 6 & 66,8 & $25^{\circ}, 8$ \\
\hline 7 & 66,0 & $25^{\circ}, 8$ \\
\hline 8 & 65,8 & $25^{\circ}, 8$ \\
\hline 9 & 65,9 & $25^{\circ}, 8$ \\
\hline 10 & 70,9 & $25^{\circ}, 9$ \\
\hline 11 & 68,5 & $25^{\circ}, 9$ \\
\hline 12 & 64,8 & $26^{\circ}$ \\
\hline 13 & 54,3 & $26^{\circ}$ \\
\hline 14 & 57,8 & $25^{\circ}, 9$ \\
\hline 15 & 61,0 & $26^{\circ}$ \\
\hline 16 & 53,2 & $26^{\circ}$ \\
\hline
\end{tabular}


Otros puntos medidos - Puntos en los bordes laterales

\begin{tabular}{|c|c|c|}
\hline PUNTO & ILUMNACION & TEMPERATURA \\
\hline $\mathrm{X}_{1}$ & 18,6 & $26^{\circ}, 1$ \\
\hline $\mathrm{X}_{2}$ & 29,8 & $26^{\circ}, 1$ \\
\hline $\mathrm{X}_{3}$ & 30,1 & $26^{\circ}$ \\
\hline $\mathrm{X}_{4}$ & 19,3 & $26^{\circ}, 1$ \\
\hline $\mathrm{X}_{3}$ & 26,4 & $26^{\circ}, 2$ \\
\hline $\mathrm{X}_{6}$ & 33,8 & $26^{\circ}, 1$ \\
\hline $\mathrm{X}_{7}$ & 33,9 & $26^{\circ}, 2$ \\
\hline $\mathrm{X}_{8}$ & 29,6 & $26^{\circ}, 1$ \\
\hline
\end{tabular}

Puntos en la segunda hilera entre los otros puntos medidos y debajo de los ventiladores

\begin{tabular}{|c|c|c|}
\hline PUNTO & ILUMINACION & TEMPERATURA \\
\hline$Y_{1}$ & 50,2 & $26^{\circ}$ \\
\hline$Y_{2}$ & 47,7 & $26^{\circ}, 1$ \\
\hline$Y_{3}$ & 48,2 & $26^{\circ}, 1$ \\
\hline
\end{tabular}

Aula pequeña - Fecha 28/10/2915 - Hora: 20 hs

Ventanas cerradas y cortinas cerradas. Sin ventiladores. Aire acondicionado apagado

\begin{tabular}{|c|c|c|}
\hline PUNTO & ILUMINACION & TEMPERATURA \\
\hline 1 & 42,4 & $24^{\circ}, 1$ \\
\hline 2 & 55,6 & $24^{\circ}, 4$ \\
\hline 3 & 46,0 & $24^{\circ}, 5$ \\
\hline 4 & 59,4 & $24^{\circ}, 8$ \\
\hline 5 & 72,7 & $24^{\circ}, 8$ \\
\hline 6 & 55,0 & $24^{\circ}, 7$ \\
\hline 7 & 46,6 & $24^{\circ}, 8$ \\
\hline 8 & 58,3 & $24^{\circ}, 8$ \\
\hline 9 & 46,5 & \\
\hline
\end{tabular}


Aula 2 - Fecha 29/10/2915

Hora de inicio: $12 \mathrm{hs}$. - Hora de finalización: $12 \mathrm{hs} 30 \mathrm{~m}$ - Soleado

- Ventiladores prendidos

- Cortinas abiertas (luz natural y artificial)

- Puerta delantera abierta.

\begin{tabular}{|c|c|c|c|}
\hline PUNTO & IIUMINACION & TEMPERATURA & VIENTO \\
\hline 1 & 62,6 & $27^{\circ}$ & 0,2 \\
\hline 2 & 52,2 & $27^{\circ}, 6$ & 0 \\
\hline 3 & 58,3 & $27^{\circ}, 9$ & 0 \\
\hline 4 & 62,2 & $27^{\circ}, 6$ & 0 \\
\hline 5 & 72,4 & $27^{\circ}, 7$ & 0,2 \\
\hline 6 & 69,0 & $27^{\circ}, 6$ & 0,7 \\
\hline 7 & 66,0 & $27^{\circ} .7$ & 0 \\
\hline 8 & 69,1 & $27^{\circ}, 6$ & 0,4 \\
\hline 9 & 67,4 & $27^{\circ}, 5$ & 0,7 \\
\hline 10 & 71,3 & $27^{\circ}, 5$ & 0,7 \\
\hline 11 & 68,7 & $27^{\circ}, 5$ & 0,9 \\
\hline 12 & 70,2 & $27^{\circ}, 5$ & 0 \\
\hline 13 & 60,5 & $27^{\circ}, 3$ & 0 \\
\hline 14 & 59,7 & $27^{\circ}, 3$ & 0 \\
\hline 15 & 61,9 & $27^{\circ}, 3$ & 0,6 \\
\hline 16 & 57,0 & $27^{\circ}, 4$ & 0 \\
\hline
\end{tabular}

Puntos en los laterales: sobre la pared de las ventanas y la de las puertas:

\begin{tabular}{|c|c|c|c|}
\hline PUNTO & ILUMINACION & TEMPERATURA & VIENTO \\
\hline $\mathrm{X}_{1}$ & 49,0 & $27^{\circ}, 6$ & 0 \\
\hline $\mathrm{X}_{2}$ & 29,7 & $27^{\circ}, 3$ & 0 \\
\hline $\mathrm{X}_{3}$ & 29,2 & $27^{\circ}, 3$ & 0 \\
\hline
\end{tabular}




\begin{tabular}{|c|c|c|c|}
\hline $\mathrm{X}_{4}$ & 21,8 & $27^{\circ}, 6$ & 0 \\
\hline $\mathrm{X}_{5}$ & 45,1 & $27^{\circ}, 2$ & 0 \\
\hline $\mathrm{X}_{6}$ & 42,7 & $27^{\circ}, 3$ & 0,6 \\
\hline $\mathrm{X}_{7}$ & 39,7 & $27^{\circ}, 6$ & 0 \\
\hline $\mathrm{X}_{8}$ & 39,0 & $27^{\circ}, 7$ & 0 \\
\hline
\end{tabular}

Puntos intermedios debajo de los ventiladores y en medio de las luces

\begin{tabular}{|c|c|c|c|}
\hline PUNTO & IUMINACION & TEMPERATURA & VIENTO \\
\hline $\mathrm{Y}_{1}$ & 59 & $27^{\circ}, 5$ & 0,2 \\
\hline $\mathrm{Y}_{2}$ & 50,9 & $27^{\circ}, 2$ & 0,6 \\
\hline $\mathrm{Y}_{3}$ & 52,4 & $27^{\circ}, 2$ & 1,1 \\
\hline
\end{tabular}

(Buen ventilador)

Aulita - Fecha 30/10/2915 - Hora 12

- Cortinas corridas (Luz natural y artificial)

- Nublado

- Ventanas cerradas sin ventilador ni aire acondicionado.

\begin{tabular}{|c|c|c|c|}
\hline PUNTO & ILUMINACION & TEMPERATURA & VIENTO \\
\hline 1 & 44,0 & $23^{\circ}, 7$ & 0 \\
\hline 2 & 57,0 & $24^{\circ}, 2$ & 0 \\
\hline 3 & 47,0 & $24^{\circ}, 3$ & 0 \\
\hline 4 & 58,3 & $24^{\circ}, 5$ & 0 \\
\hline 5 & 74,5 & $24^{\circ}, 2$ & 0 \\
\hline 6 & 55,4 & $24^{\circ}, 4$ & 0 \\
\hline 7 & 60,2 & $24^{\circ}, 1$ & 0 \\
\hline 8 & 75,9 & $24^{\circ}, 1$ & 0 \\
\hline 9 & 51,3 & $24^{\circ}, 2$ & 0 \\
\hline
\end{tabular}


Aulita - Fecha 30/10/2015 (nublado) - Hora 12 hs 30m

- Cortinas corridas y luz apagada Solo luz natural.

- Ventana abierta,

\begin{tabular}{|c|c|c|c|}
\hline PUNTO & ILUMINACION & TEMPERATURA & VIENTO \\
\hline 1 & 2,7 & $25^{\circ}, 3$ & 0 \\
\hline 2 & 2,6 & $24^{\circ}, 8$ & 0 \\
\hline 3 & 2,6 & $24^{\circ}, 7$ & 0 \\
\hline 4 & 6,2 & $23^{\circ}, 7$ & 0 \\
\hline 5 & 5,8 & $23^{\circ}, 1$ & 0 \\
\hline 6 & 5,0 & $23^{\circ}, 2$ & 0 \\
\hline 7 & 14,8 & $23^{\circ}, 7$ & 0 \\
\hline 8 & 40,2 & $23^{\circ}, 9$ & 0,2 \\
\hline 9 & 11,3 & $22^{\circ}, 8$ & 0 \\
\hline
\end{tabular}

Aula 2 - Con luz solo natural y cortinas abiertas

\begin{tabular}{|c|c|c|}
\hline PUNTO & ILUMINACION & TEMPERATURA \\
\hline 4 & 1,9 & $25^{\circ}, 2$ \\
\hline 5 & 1,5 & $25^{\circ}, 7$ \\
\hline 12 & 1,4 & $25^{\circ}, 6$ \\
\hline 13 & 1,2 & $25^{\circ}, 8$ \\
\hline 14 & 0,5 & $28^{\circ}, 4$ \\
\hline 15 & 0,3 & $26^{\circ}, 0$ \\
\hline
\end{tabular}

\section{Punto Exterior}

Fecha 29/10/2015 - Hora 12 soleado

\begin{tabular}{|c|c|c|}
\hline ILUMINACION & TEMPERATURA & VIENTO \\
\hline+2000 & $34^{\circ}, 7$ & 3,9 \\
\hline
\end{tabular}


Fecha 30/10/2015 - Hora 12 nublado

\begin{tabular}{|c|c|c|}
\hline ILUMINACION & TEMPERATURA & VIENTO \\
\hline 1922 & $20^{\circ}, 6$ & 1,4 \\
\hline
\end{tabular}

Se encontró que todos los puntos de la cuadricula estaban iluminados de acuerdo a lo establecido para un salón de clases o laboratorios. Considerando que en el salón de clases los puntos centrales de las cuadriculas coincidían con las luminarias, por lo que se midieron otros puntos testigos, los cuales apenas cumplían la normativa

\section{CONCLUSIONES:}

Los 16 puntos mínimos resultantes en la cuadricula del salón de clases coinciden con las luces es decir que se ubican exactamente debajo de las iluminarias instaladas en el aula .Por eso también se tomaron las medidas en los puntos denominados $\mathrm{Y}$ que quedan intermedios de las luces y debajo de los ventiladores.

Se constato que el grado de iluminación es el adecuado, en un día normal soleado o nublado, pero que no se podría llevar adelante una clase sin iluminación artificial, con acondicionamiento de la temperatura del aire. Se considera importante, el conocimiento de las ventanas, su orientación y vistas al exterior libres o con sombras, que permitirían mejorar la iluminación de los locales analizados

Bibliografía

-Ley 19587, Higiene y seguridad en el trabajo

-Norma IRAM AADL J 2006

- Decreto Nacional 351/79

- Guía practica sobre iluminación en el ambiente laboral. Superintendencia de Riesgos de Trabajo 